\title{
CONF-970503 --
}

\section{ERNEST DRLANDI LAWRENCE}

BERKELEY NATIONAL LABDRATIRY

\section{Laser Acceleration in \\ Vacuum with an Open \\ Iris-Loaded Waveguide}

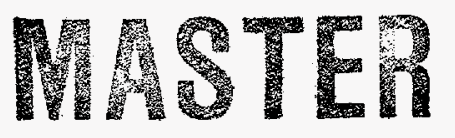

Ming Xie

Accelerator and Fusion

Research Division

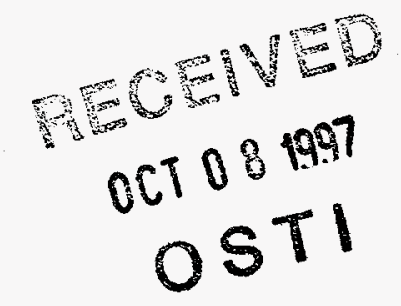

May 1997

Presented at the

1997 Particle

Accelerator Conference,

Vancouver, BC, Canada,

May 12-16, 1997,

and to be published in

the Proceedings

DATRIOUTON OF THIS DOCUMETT is URAMTED of

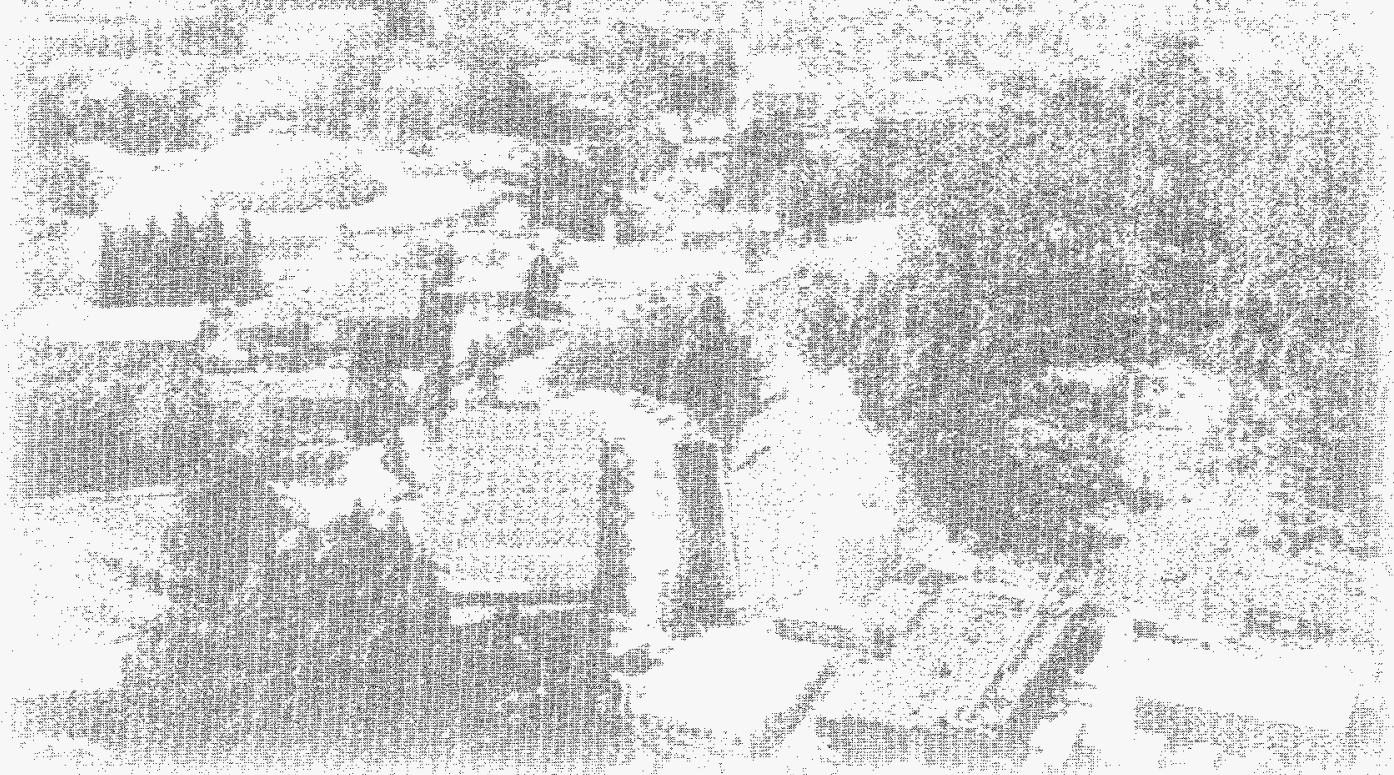




\section{DISCLAIMER}

This document was prepared as an account of work sponsored by the United States Government. While this document is believed to contain correct information, neither the United States Government nor any agency thereof, nor The Regents of the University of California, nor any of their employees, makes any warranty, express or implied, or assumes any legal responsibility for the accuracy, completeness, or usefulness of any information, apparatus, product, or process disclosed, or represents that its use would not infringe privately owned rights. Reference herein to any specific commercial product, process, or service by it: trade name, trademark, manufacturer, or otherwise, does not necessarily constitute or imply its endorsement, recommendation, or favoring by the United States Government or any agency thereof, or The Regents of the University of California. The views and opinions of authors expressed herein do not necessarily state or reflect those of the United States Government or any agency thereof, or The Regents of the University of California.

Ernest Orlando Lawrence Berkeley National Laboratory is an equal opportunity employer. 
LBNL-40249

CBP Note-219

$\mathrm{UC}-414$

\title{
Laser Acceleration in Vacuum with an Open Iris-Loaded Waveguide*
}

\author{
Ming Xie \\ Accelerator and Fusion Research Division \\ Ernest Orlando Lawrence Berkeley National Laboratory \\ Berkeley, CA 94720 USA
}

May 1997

* This work was supported by the Director, Office of Energy Research, Office of High Energy and Nuclear Physics, Division of High Energy Physics, of the U.S. Department of Energy under Contract No. DE-AC 0376 SF00098. 


\section{DISCLAMIER}

Portions of this doecoment may be illegibie in electronic image products. Images are produced from the best available original docement 


\title{
LASER ACCELERATION IN VACUUM WITH AN OPEN IRIS-LOADED WAVEGUIDE*
}

\author{
Ming Xie, Lawrence Berkeley National Laboratory, Berkeley, CA 94720, USA
}

\begin{abstract}
An open iris-loaded waveguide structure is considered for laser acceleration of highly relativistic particle in vacuum. Complete characterization of all eigenmodes are given in analytical form for the structure. In particular the dominant radially polarized TM mode is evaluated in detail for laser acceleration. The entire parameter space is searched and it is found that below the laser damage threshold of the structure an acceleration gradient around $1 \mathrm{GV} / \mathrm{m}$ can be obtained over a phase slippage length of $10 \mathrm{~s}$ of $\mathrm{cm}$ with TWs laser in the wavelength range from 1 to $10 \mu \mathrm{m}$.
\end{abstract}

\section{INTRODUCTION}

The Open Iris-Loaded waveguide Structure (OILS) considered in this paper is made of a series of equally spaced thin screens each having a circular aperture. It differs from the usual iris-loaded linac structure in the following aspects: first of all it does not have side walls thus called an open structure; secondly all its characteristic dimensions i.e., radius of the aperture (a) and separation between the adjacent apertures $(\mathrm{L})$, are much larger than laser wavelength $(\lambda)$. As a result the eigenmodes of the structure are determined dominantly by diffraction. Recently, Pantell [1] presented a calculation of the longitudinal field component in the structure using the numerical method of Fox-Li [2] and discussed the possibility of laser acceleration. But, soon after that Pantell [3] claimed that an additional fast axial phase oscillation absent from the Fox-Li's original calculations [2] was found and thus concluded that the structure is not suitable for laser acceleration.

However the claimed fast phase oscillation does not exist. The numerical method of Fox- $\mathrm{Li}$ is not an appropriate approach to this problem. To demonstrate net energy gain for a test particle, the field everywhere along the trajectory needs to be calculated. The kernel of the Fresnel integral used in Fox-Li's method becomes increasingly fast oscillatory for the field at locations close to the diffracting aperture, causing the integral extremely cumbersome to calculate and prone to various sources of numerical and systematic errors. To avoid the numerical difficulties fully analytical approach is taken in this paper.

\section{EIGENMODES OF THE OPEN WAVEGUIDE}

Starting from source free Maxwell Equations in vacuum, we seek axially symmetric, radially polarized TM mode of the form: $\mathbf{E}(\mathbf{r}, \mathrm{z}, \mathrm{t})=\mathbf{E}_{\mathbf{r}}+\mathbf{E}_{\mathrm{z}}, \mathbf{H}(\mathrm{r}, \mathrm{z}, \mathrm{t})=\mathbf{H}_{\varphi}$. All

* This work was supported by the U.S. Department of Energy under contract No. DE-AC03-76SF00098. other types of modes can be obtained following the same procedures. For fields with $\exp (-i \omega t)$ dependence, each component satisfies the scalar Helmholtz wave equation, $\nabla^{2} \Phi+\mathrm{k}^{2} \Phi=0$, where $\mathrm{k}=\omega / \mathrm{c}$. Under the conditions $\mathrm{L} \gg \lambda$ and $\mathrm{a} \gg \lambda$, the wave equation can be further reduced to a parabolic equation

$$
\nabla_{t}^{2} \Psi+2 \mathrm{ik} \frac{\partial \Psi}{\partial \mathrm{z}}=0
$$

where $\Phi(\mathrm{r}, \mathrm{z})=\Psi(\mathrm{r}, \mathrm{z}) \exp (\mathrm{ikz}), \Psi(\mathrm{r}, \mathrm{z})$ is the envelope slowly varying in $\mathrm{z}$, and $\nabla_{t}^{2}$ is the transverse Laplacian.

For open waveguide without side walls it is convenient to solve first for the radial component. With $\Phi=E_{\mathrm{r}}(r, z)$, Eq.(1) becomes

$$
\frac{\partial^{2} \Psi}{\partial \mathrm{r}^{2}}+\frac{1}{\mathrm{r}} \frac{\partial \Psi}{\partial \mathrm{r}}-\frac{1}{\mathrm{r}^{2}} \Psi+2 \mathrm{ik} \frac{\partial \Psi}{\partial \mathrm{z}}=0 .
$$

This is the equation with azimuthal mode number $m=1$, and its solution may be labeled as $\Psi_{1 n}^{(r)}(r, z)$, where $n$ is the radial mode index, and the superscript indicates the mode is radially polarized. The radially polarized mode is a linear superposition of two degenerate, orthogonal linearly polarized modes $\Psi_{1 \mathrm{n}}^{(\mathrm{x})}(\mathrm{r}, \mathrm{z}) \sin (\varphi)$ and $\Psi_{1 \mathrm{n}}^{(y)}(\mathrm{r}, \mathrm{z}) \cos (\varphi)$.

Our goal is to solve Eq.(2) for the eigenmodes in an OILS. This problem is shown to be equivalent to finding the eigenmodes in a Fabry-Perot optical resonator with two circular plane mirrors of radius a, separated by a distance L [2]. Under appropriate boundary conditions, Vainshtein [4] obtained solution of the eigenmodes to all orders inside such a resonator. Once $E_{\mathrm{r}}$ is solved, all other field components can be determined directly through Maxwell equations. With all spatial-temporal dependence explicitly included, the solution reads:

$$
\begin{gathered}
E_{z}=E_{a} J_{0}\left(k_{r} r\right) \exp \left[i\left(k_{z} z-\omega t\right)\right], \\
E_{r}=-i\left(k_{z} / k_{r}\right) E_{a} J_{1}\left(k_{r} r\right) \exp \left[i\left(k_{z} z-\omega t\right)\right], \\
H_{\varphi}=E_{r} / Z_{T M}, \\
k_{r}=\frac{\nu_{1 n}}{a[1+\eta(1+i) / M]}, \quad k_{z}=k-k_{r}^{2} / 2 k
\end{gathered}
$$

where $\mathrm{M}=\sqrt{8 \pi \mathrm{N}}, \mathrm{N}$ is Fresnel number defined as $\mathrm{N}=$ $\mathrm{a}^{2} / \lambda \mathrm{L}, \eta=-\zeta(1 / 2) / \sqrt{\pi} \approx 0.824, \zeta(\mathrm{z})$ is Riemann's Zeta function, $\mathrm{Z}_{\mathrm{TM}}=\left(\mathrm{k}_{\mathrm{z}} / \mathrm{k}\right) \mathrm{Z}_{0}, \mathrm{Z}_{\mathrm{TM}}$ is the impedance of TM wave, $Z_{0}$ is the vacuum impedance, $\nu_{1 n}$ is the $n$th zero of Bessel function satisfying $\mathrm{J}_{1}\left(\nu_{1 \mathrm{n}}\right)=0$, and $\mathrm{E}_{\mathrm{a}}$ is peak acceleration gradient. It should be noted that as a result of slowly varying envelope approximation, the modes are characterized by, $\left|k_{z}\right| \sim k$ and $\left|k_{r}\right| \ll k$. The solution is more accurate at small diffraction loss per cell, or equivalently when $N \gg 1$, which happens to be the regime favorable for laser acceleration, as will be discussed later. 


\section{ACCELERATOR CONSIDERATIONS}

In this section, we exploit properties of the $\mathrm{TM}_{1 \mathrm{n}}^{(\mathrm{r})}$ mode, and derive necessary formulas and scaling laws for the evaluation of OILS as an acceleration structure.

\subsection{General Characteristics and Formulas}

For convenience, let's introduce a complex quantity $\alpha \equiv$ $\alpha_{\mathrm{r}}+\mathrm{i} \alpha_{\mathrm{i}}=\mathrm{ik} \mathrm{r}_{\mathrm{r}}^{2} / 2 \mathrm{k}$, and from Eq.(6) we have

$$
\begin{aligned}
& \alpha_{\mathrm{r}}=\frac{4 \nu_{\mathrm{ln}}^{2} \eta(\mathrm{M}+\eta)}{\mathrm{L}\left[(\mathrm{M}+\eta)^{2}+\eta^{2}\right]^{2}}, \\
& \alpha_{\mathrm{i}}=\frac{2 \nu_{\ln }^{2} \mathrm{M}(\mathrm{M}+2 \eta)}{\mathrm{L}\left[(\mathrm{M}+\eta)^{2}+\eta^{2}\right]^{2}} .
\end{aligned}
$$

The $\{z, t\}$ dependence for all field components becomes

$$
\left\{E_{z}, E_{r}, H_{\varphi}\right\} \sim e^{i\left[\left(k-\alpha_{i}\right) z-\omega t\right]-\alpha_{x} z},
$$

and power flow through the waveguide is simply

$$
\begin{gathered}
\mathrm{P}(\mathrm{z})=\mathrm{P}_{0} \mathrm{e}^{-2 \alpha_{\mathrm{r}} \mathrm{z}}, \\
\mathrm{P}_{0}=\frac{\pi \mathrm{a}^{2}\left|\mathrm{k}_{\mathrm{z}} / \mathrm{k}_{\mathrm{r}}\right|^{2} \mathrm{E}_{\mathrm{a}}^{2}}{\operatorname{Re}\left(\mathrm{Z}_{\mathrm{TM}}\right)} \int_{0}^{1}\left|\mathrm{~J}_{1}\left(\mathrm{k}_{\mathrm{r}} \mathrm{a} \rho\right)\right|^{2} \rho \mathrm{d} \rho .
\end{gathered}
$$

As seen from Eqs. $(9,10)$, the mode is characterized by a phase velocity, $v_{p}=\omega /\left(k-\alpha_{i}\right)$, larger than $c$, and a power attenuation due to diffraction loss at the apertures. However, for sufficiently large $N$ both phase slippage per cell, $\phi_{\mathrm{c}}=\alpha_{\mathrm{i}} \mathrm{L}$, and fractional power loss per cell, $\alpha_{\mathrm{c}}=2 \alpha_{\mathrm{r}} \mathrm{L}$, can be made as small as desired, as seen from Eqs. $(7,8)$. Let's define a slippage length, $L_{s}=\pi / \alpha_{i}$, over which an acceleration phase slippage of $\pi$ is experienced by a highly relativistic particle $(v \approx c)$ moving along the $z$-axis. The energy gain of the test particle traversing a slippage length thus can be calculated as

$$
\Delta W_{s}=e E_{a} \int_{0}^{L_{s}} \sin \left(\alpha_{i} z\right) e^{-\alpha_{r} z} d z=e E_{a} L_{s} T_{s},
$$

where $T_{s}$ is a reduction factor due to a full $\pi$ phase slippage and the attenuation of the acceleration field over a slippage length, given by

$$
\mathrm{T}_{\mathrm{s}}=\frac{1+\mathrm{e}^{-\left(\alpha_{\mathrm{r}} / \alpha_{\mathrm{i}}\right) \pi}}{\pi\left[1+\left(\alpha_{\mathrm{r}} / \alpha_{\mathrm{i}}\right)^{2}\right]}
$$

Two other figures of merit, the shunt impedance per unit length $\mathrm{Z}_{\mathrm{L}}$ and the $Q$ factor, are given here for comparison with traditional microwave acceleration structures

$$
\begin{gathered}
\mathrm{Z}_{\mathrm{L}}=\frac{\left|\mathrm{E}_{\mathrm{z}}(\mathrm{r}=0, \mathrm{z})\right|^{2}}{-\mathrm{dP} / \mathrm{dz}}=\frac{\mathrm{E}_{\mathrm{a}}^{2}}{2 \alpha_{\mathrm{r}} \mathrm{P}_{0}}, \\
\mathrm{Q}=\frac{\omega \mathrm{U}}{-\mathrm{dP} / \mathrm{dz}}=\frac{\pi}{\lambda \alpha_{\mathrm{r}}\left(\mathrm{v}_{\mathrm{g}} / \mathrm{c}\right)} \approx \frac{\pi}{\lambda \alpha_{\mathrm{r}}},
\end{gathered}
$$

where $U$ is the field energy per unit length given by $U=$ $P / v_{g}$, and $v_{g}$ is the group velocity very close to $c$.

Another important concern for high gradient acceleration by a waveguide is surface field on the structure which is limited by damage threshold. For OILS, we define an edge field by $E_{e}=\left|E_{r}(r=a, z=0)\right|$, and it can be shown that the condition $E_{e}>E_{a}$ always holds.

\subsection{Weak Diffraction Limit and Scaling Laws}

Before going to the exploration of OILS performance over design parameter space using the general formulas provided in Sec.(3.1), it is instructive to look at more transparent weak diffraction limit and scaling laws for several important performance parameters. We will consider the dominant radial mode with $\mathrm{n}=1$ and $\nu_{11}=3.832$. Keeping only the leading term while taking the limit $N \gg 1$, we have

$$
\begin{gathered}
\frac{\mathrm{E}_{\mathrm{a}}}{\mathrm{E}_{\mathrm{e}}} \rightarrow \frac{\sqrt{\mathrm{N}}(\lambda / \mathrm{a})}{\sqrt{\pi} \eta\left|\mathrm{J}_{0}\left(\nu_{11}\right)\right|} \approx 1.7 \sqrt{\mathrm{N}}(\lambda / \mathrm{a}) \\
\mathrm{L}_{\mathrm{s}} \longrightarrow \frac{4 \pi^{2} \mathrm{a}^{2}}{\nu_{11}^{2} \lambda} \approx 2.7 \mathrm{a}^{2} / \lambda=2.7 \mathrm{~N} \mathrm{~L} \\
\alpha_{\mathrm{s}} \longrightarrow 1-\mathrm{e}^{-\eta \sqrt{2 \pi / \mathrm{N}}} \approx 1-\mathrm{e}^{-2.1 / \sqrt{\mathrm{N}}}, \\
\Delta \mathrm{W}_{\mathrm{s}}[\mathrm{MeV}] \longrightarrow 2.9 \sqrt{\mathrm{N}} \mathrm{a}[\mathrm{mm}] \mathrm{E}_{\mathrm{e}}[\mathrm{GV} / \mathrm{m}] \\
\mathrm{I}_{\mathrm{av}}\left[\mathrm{PW} / \mathrm{cm}^{2}\right] \longrightarrow 1.7 \times 10^{-4} \mathrm{NE}_{\mathrm{e}}^{2}[\mathrm{GV} / \mathrm{m}] \\
\frac{\mathrm{I}_{\mathrm{e}}}{\mathrm{I}_{\mathrm{av}}} \longrightarrow \frac{\left(\nu_{11} \eta\right)^{2}}{4 \pi \mathrm{N}} \approx 0.79 / \mathrm{N},
\end{gathered}
$$

where $\alpha_{s}$ is the fractional power loss per slippage length, $I_{\text {av }}$ is the laser intensity averaged over waveguide cross section at $z=0$, thus the required laser power is given by $P_{0}=\pi a^{2} I_{a v}$, and $I_{e}$ is the laser intensity at the edge of aperture. Also we have $\mathrm{T}_{\mathrm{s}} \rightarrow 2 / \pi$, and with $\Gamma=\mathrm{k}-\alpha_{\mathrm{i}}$,

$$
\begin{aligned}
& \mathrm{v}_{\mathrm{p}}=\frac{\omega}{\Gamma} \approx \frac{\mathrm{c}}{1-\frac{1}{2}\left(\frac{\nu_{11} \lambda}{2 \pi \mathrm{a}}\right)^{2}}, \\
& \mathrm{v}_{\mathrm{g}}=\frac{\mathrm{d} \omega}{\mathrm{d} \Gamma} \approx \frac{\mathrm{c}}{1+\frac{1}{2}\left(\frac{\nu_{11} \lambda}{2 \pi \mathrm{a}}\right)^{2}} .
\end{aligned}
$$

The most important characteristics of OILS as a potentially attractive structure for laser acceleration is revealed by Eq.(16). It is shown that the ratio of acceleration to edge (surface) field is enhanced from the usual scaling for all near field accelerations [5], $\lambda / a$, by a large factor, $\sqrt{\mathrm{N}}$. As a result, substantial acceleration gradient can be obtained on the axis with a favorable intensity scaling, Eq.(21), even though the boundary is hundreds of wavelengths away.

\section{PERFORMANCE EVALUATION}

With the parameter definitions and general formulas given in Sec.(3.1), we are now ready to evaluate OILS performance. It is noted that there are only four independent parameters and they are chosen for convenience to be $\left\{\lambda, E_{e}, a / \lambda, N\right\}$. We are interested in only a few discrete 
wavelengths where bright sources are available, in particular at 1 and $10 \mu \mathrm{m}$. The maximum tolerable edge field $\mathrm{E}_{\mathrm{e}}$ is determined by laser damage threshold, which can often be set at a constant value given laser wavelength and material of the structure. It can be inferred from the experimental data [6] that $10 \mathrm{GV} / \mathrm{m}$ seems to be a reasonable upper limit for $E_{\mathrm{e}}$ at $\lambda=1 \mu \mathrm{m}$. Thus we are left with only two independent parameters $\{a / \lambda, N\}$ to vary, all dependent parameters can therefore be conveniently visualized in contour plots.

Shown in Fig.(1-3) are the peak acceleration gradient, energy gain per slippage length, and the required laser power, respectively, for $\left\{\lambda=1 \mu \mathrm{m}, E_{\mathrm{e}}=10 \mathrm{GV} / \mathrm{m}\right\}$. Four examples including $\left\{\lambda=10 \mu \mathrm{m}, E_{\mathrm{e}}=5 \mathrm{GV} / \mathrm{m}\right\}$ cases with more complete listing of performance parameters are given in Table 1. All these results can be readily scaled to other parameter regime of interest according to the scaling laws given in Sec.(3.2).

Table 1. Example Cases of Acceleration Parameters

\begin{tabular}{|c|c|c|c|c|}
\hline CASES & IA & IB & IIA & IIB \\
\hline$\lambda(\mu \mathrm{m})$ & 1 & 1 & 10 & 10 \\
\hline $\mathrm{a}(\mathrm{mm})$ & 0.5 & 0.25 & 1 & 0.5 \\
\hline $\mathrm{L}(\mathrm{mm})$ & 1 & 0.125 & 2 & 1 \\
\hline $\mathrm{N}$ & 250 & 500 & 50 & 25 \\
\hline $\mathrm{E}_{\mathrm{a}}(\mathrm{GV} / \mathrm{m})$ & 0.54 & 1.5 & 0.6 & 0.84 \\
\hline$\Delta \mathrm{W}_{\mathrm{s}}(\mathrm{MeV})$ & 227 & 161 & 100 & 35 \\
\hline $\mathrm{L}_{\mathrm{s}}(\mathrm{cm})$ & 69 & 17 & 28 & 7.2 \\
\hline $\mathrm{P}_{0}(\mathrm{TW})$ & 34 & 17 & 7.1 & 0.92 \\
\hline $\mathrm{I}_{\mathrm{av}}\left(\mathrm{PW} / \mathrm{cm}^{2}\right)$ & 4.3 & 8.6 & 0.23 & 0.12 \\
\hline $\mathrm{I}_{\mathrm{e}}\left(\mathrm{TW} / \mathrm{cm}^{2}\right)$ & 13 & 13 & 3.3 & 3.3 \\
\hline$\phi_{\mathrm{c}}(\mathrm{degree})$ & 0.26 & 0.13 & 1.3 & 2.5 \\
\hline$\alpha_{\mathrm{c}}(\%)$ & 0.019 & 0.0067 & 0.2 & 0.56 \\
\hline$\alpha_{\mathrm{s}}(\%)$ & 12 & 8.8 & 25 & 33 \\
\hline $\mathrm{Q}\left(10^{6}\right)$ & 33 & 12 & 0.62 & 0.11 \\
\hline $\mathrm{Z}_{\mathrm{L}}(\mathrm{M} \Omega / \mathrm{m})$ & 0.045 & 0.25 & 0.049 & 0.14 \\
\hline $\mathrm{T}_{\mathrm{s}}$ & 0.62 & 0.62 & 0.59 & 0.58 \\
\hline $\mathrm{E}_{\mathrm{e}}(\mathrm{GV} / \mathrm{m})$ & 10 & 10 & 5 & 5 \\
\hline
\end{tabular}

\section{CONCLUSIONS}

I have presented a systematic evaluation of laser acceleration inside an OILS assuming well established eigenmode. Analysis of planar and rectangular structures can be carried out parallel to what's done here. The scaling laws derived from the powerful analytical approach of Vainshtein have both revealed the simplicity of the acceleration mechanism and uncovered some surprisingly favorable characteristics of such a diffraction dominated structure. However I have not touched upon such critical issues as wakefield, beam loading, and ways to couple laser power in and out of the structure without significantly degrading acceleration performance. But, I do want to point out in passing that the axicon scheme used to generate radially polarized laser beam for the inverse Cherenkov laser acceleration experiment [7] appears to be an interesting candidate for OILS mode injection. Further investigation on this and other critical issues will be presented in a forthcoming paper.

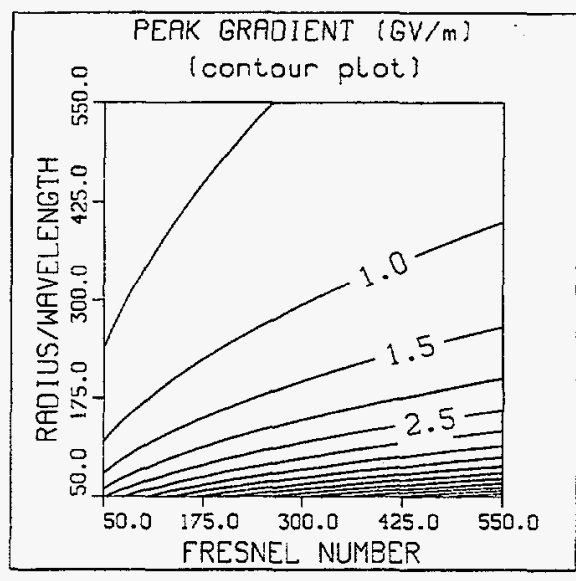

Figure 1: $\mathrm{E}_{\mathrm{a}}$ vs. $\{\mathrm{N}, \mathrm{a} / \lambda\} @ \lambda=1 \mu \mathrm{m}, \mathrm{E}_{\mathrm{e}}=10 \mathrm{GV} / \mathrm{m}$.

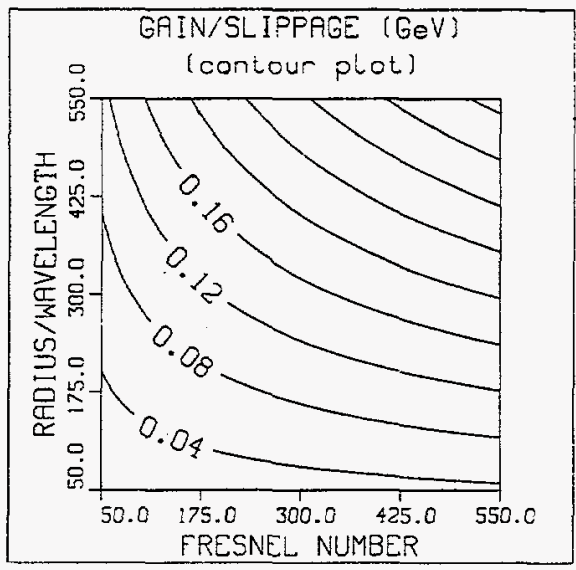

Figure 2: $\Delta \mathrm{W}_{\mathrm{s}}$ vs. $\{\mathrm{N}, \mathrm{a} / \lambda\} @ \lambda=1 \mu \mathrm{m}, \mathrm{E}_{\mathrm{e}}=10 \mathrm{GV} / \mathrm{m}$.

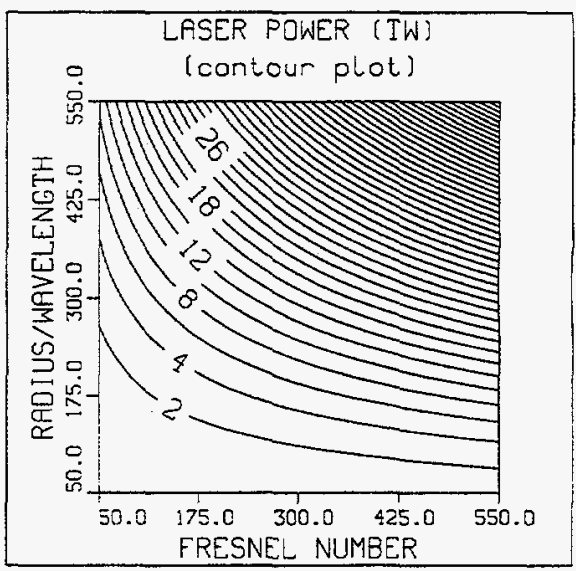

Figure 3: $P_{0}$ vs. $\{\mathrm{N}, \mathrm{a} / \lambda\} @ \lambda=1 \mu \mathrm{m}, \mathrm{E}_{\mathrm{e}}=10 \mathrm{GV} / \mathrm{m}$.

\section{REFERENCES}

[1] R. Pantell, talk presented at FEL conference, August, 1996.

[2] A. G. Fox and T. Li, Bell System Technical J., 40(1961)453.

[3] R. Pantell, private comm. at AAC workshop, October, 1996.

[4] L. A. Vainshtein, Soviet Physics JETP, 17(1963)709.

[5] R. B. Palmer, AIP Conference Proceedings, 335(1995)90.

[6] B. C. Stuart, el al., Phys. Rev. Lett., 74(1995)2248.

[7] W. D. Kimura, et al., Phys. Rev. Lett., 74(1995)546. 